\title{
sciendo
}

\author{
BULGARIAN ACADEMY OF SCIENCES
}

CYBERNETICS AND INFORMATION TECHNOLOGIES • Volume 21, No 2

Sofia $2021 \quad$ Print ISSN: 1311-9702; Online ISSN: 1314-4081

DOI: $10.2478 /$ cait-2021-0021

\section{A New Digital Image Steganography Based on Center Embedded Pixel Positioning}

\author{
Dedi Darwis ${ }^{1,2}$, Akmal Junaidi ${ }^{3}$, Dewi Asiah Shofiana ${ }^{3}$, Wamiliana ${ }^{4}$ \\ ${ }^{1}$ Doctoral at Faculty of Mathematics and Natural Science, Universitas Lampung, Lampung, Indonesia \\ ${ }^{2}$ Faculty of Engineering and Computer Science, Universitas Teknokrat Indonesia, Lampung, Indonesia \\ ${ }^{3}$ Department of Computer Science, Universitas Lampung, Lampung, Indonesia \\ ${ }^{4}$ Department of Mathematics, Universitas Lampung, Lampung, Indonesia \\ E-mails:_darwisdedi@teknokrat.ac.id akmal.junaidi@fmipa.unila.ac.id \\ dewi.asiah@fmipa.unila.ac.id wamiliana.1963@fmipa.unila.ac.id
}

\begin{abstract}
In this study we propose a new approach to tackle the cropping problem in steganography which is called Center Embedded Pixel Positioning (CEPP) which is based on Least Significant Bit (LSB) Matching by setting the secret image in the center of the cover image. The evaluation of the experiment indicated that the secret image can be retrieved by a maximum of total $40 \%$ sequential cropping on the left, right, up, and bottom of the cover image. The secret image also can be retrieved if the total asymmetric cropping area is $25 \%$ that covered two sides (either left-right, left-up or right-up). In addition, the secret image can also be retrieved if the total asymmetric cropping area is $70 \%$ if the bottom part is included. If asymmetric cropping area included three sides, then the algorithm fails to retrieve the secret image. For cropping in the botom the secret image can be extracted up to $70 \%$.
\end{abstract}

Keywords: Cover image, cropping, security, stego image, steganography.

\section{Introduction}

Steganography is a technical art on how to hide messages into other media, such as image, text, audio, and video, that are all known as the steganographic cover. Steganographic cover contains secret images in which can only be extracted by the recipient. All digital files in bits can be the media of steganography [1-3].

Secret images in this research are images, whereas RGB (Red, Green, Blue) images in Portable Network Graphics (PNG) format, which is a lossless compression image format, were used as the media. The algorithm's reliability determines the image quality resulting from steganography in the embedding and extraction process $[4,5]$. There are two primary methods to test the image quality:

1. Fidelity, the ability to accurately process the image with no visual distortion nor information loss by calculating the Peak-Signal to Noise-Ration (PSNR) [6, 7]; 
2. Robustness, the stego-analysis attack or image manipulation: attacking the stego-image with image processing such as crop, blur, noise, rotate, etc., [8-10].

Image lacks the ability to preserve information when a stego-analysis attack occurs, making robustness as the main concern in steganography [11, 12]. Cropping is a type of image manipulation that will effectively distort the value of image pixels, causing the hidden message in a stego-image to get corrupted $[6,13]$. The crop manipulation is effective since secret images are generally stored in a stego-image at the very last bit of the image located in the top-left corner, which can be cropped easily $[14,15]$.

Least Significant Bit (LSB) is a common technique in steganography that can insert secret images to an image with no visual difference between the original image and stego-image $[16,17]$. The LSB method has been developed by implementing Nine-Pixel Differencing and LSB Substitution that can increase the embedding capacity while maintaining the image imperceptible as well as improving the fidelity value. This development is performed by modifying LSB bit of the image into a $3 \times 3$ block using the equation $d=\frac{1}{8} \sum_{i=0}^{8}\left|x_{i}-x_{\min }\right|$. The experiment shows that, on average, the Mean Square Error (MSE) and PSNR increases by -0.5375 and 47 sequentially, with the average bit storage capacity about 187.069. However, this method is still unable to resist the image processing attack $[18,19]$.

A work by A 1-A f a n d y et al. [3] discusses a conceptual framework to preserve the lost information from a cropped stego-image. Crop manipulation effectively attacks the stego-image since hidden information is usually stored in the last bit, located at the corner of the image. Hence, when the image is cropped, information extraction will be difficult or even impossible [3].

Realizing that cropping manipulation mostly fail to retrieve secret image if cropping is done on the left above position, a new approach using Center Sequential Technique (CST) is proposed to overcome this problem. In CST, the cover image and the secret image (in this method the used image also as the secret image), were grayscale type [14]. In CST, to determine the center of the cover image the length and the width both were divided by two.

In this research we enhanced the CST method by allowing RGB for cover image and secret image (still use image as the secret image), and refine the method for embedding process, and we called the method as Center Embedded Pixel Positioning (CEPP).

\section{CEPP method}

This work has develops a technique to insert secret images sequentially by embedding the message image in the form of bits into the center position of cover image by calculating the length and width of the image. The method is named CEPP since the hidden message is embedded at the center of the cover image by developing the way in which the pixel sequential works. In general, the process steps of CEPP are as follows. 


\subsection{Message embedding method}

PNG image is utilized as the hidden message that will be embedded into the cover image in the same format. Fig. 1 illustrates the general message insertion process in the proposed method.

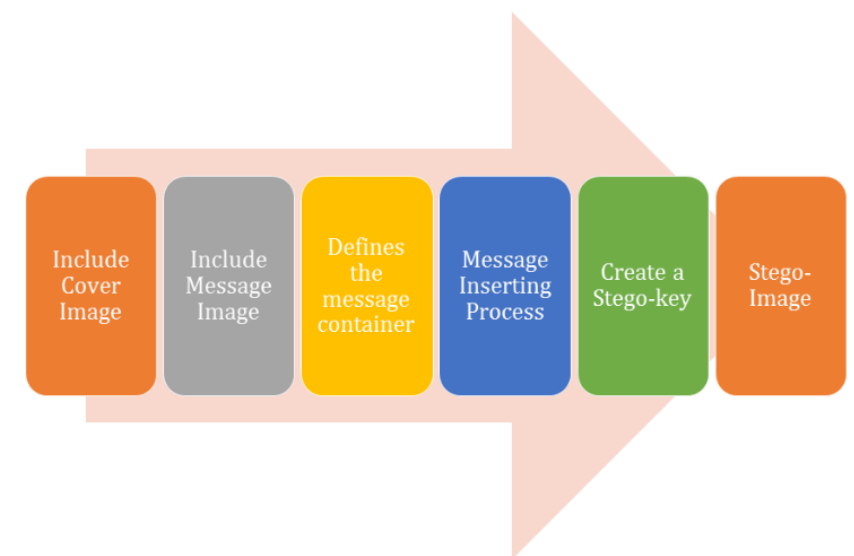

Fig. 1. Message inserting process

The procedure below describes the steps of the embedding/inserting process.

1. Include cover image

In this step, the algorithm scans the number of columns of the cover image. If the number of columns $=a \bmod (8)$, then $a$ is the number of shifting needed to put the initial point (north west corner) of the message container area.

2. Include message image

The size of the secret image must be smaller than the cover image. In this proposed algorithm, we implement the secret images which size do not exceed 110 pixels. The reason for using 110 pixels is due to the quality of the stego-image. In this step pixels value is converted to binary and then change the last bit of the image to be "O" using LSB.

3. Define the message container area

This process is at the heart of the approach to the proposed method. The first step carried out was to change the pixel value of the message image into a list form, then validates the width and height of the cover image so that it is divisible by 8 . Afterward, determine the coordinates to insert the message (xs, xe, ys, ye), called the container, as illustrated in Fig. 2. Calculation on how to determine the container coordinate is provided in

$$
\begin{aligned}
& \mathrm{xs}=\frac{1}{4} w, \quad \mathrm{xe}=w-\mathrm{xs}, \\
& \mathrm{ys}=\frac{1}{4} h, \quad \mathrm{ye}=h-\mathrm{ys},
\end{aligned}
$$

where: $x s=$ Container's width start point; $x e=$ Container's width end point; ys $=$ Container's height start point; ye $=$ Container's height end point; $w=$ Cover image width; $h=$ Cover image height . 


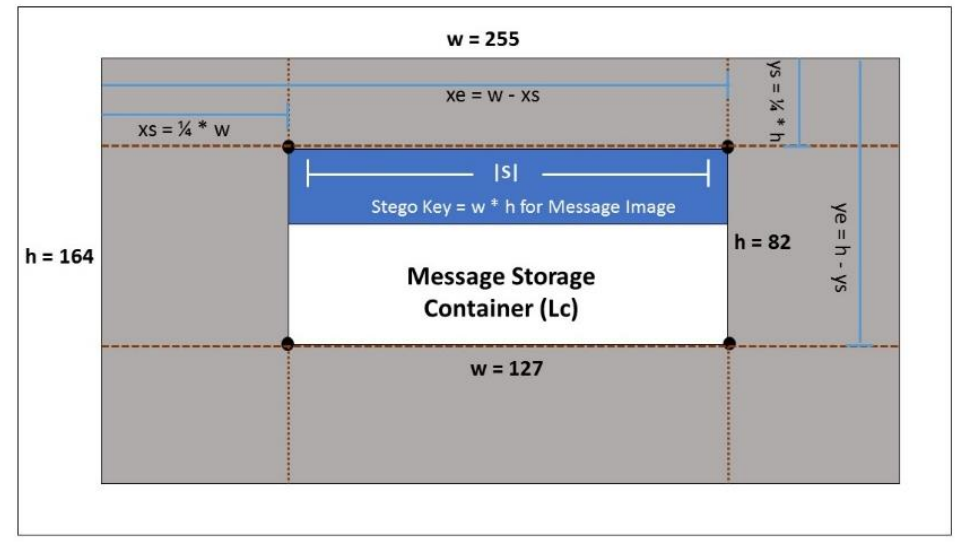

Fig. 2. Preparation of container

After obtaining the size of the message container, validation is performed to ensure that the width of the container is divisible by 8 , as in (3) as follows:

$$
\begin{aligned}
& \mathrm{xs}=\mathrm{xs}+(8-\mathrm{xs} \bmod (8)), \\
& \mathrm{xe}=\mathrm{xe}+(8-\mathrm{xe} \bmod (8)) .
\end{aligned}
$$

If the message length is greater or equal to the area of the container, then the message cannot be embedded. Otherwise, all message bits are embedded in the specified container, and a stego-image is created.

4. Message inserting process

This process starts by changing the pixel value of the cover image into binary and then changing all the last bits of the cover image into "1" using LSB. This changing is needed to discriminate message counter area and other areas. Since the image is in RGB then this procedure should be done for each channel (Red, Green, Blue). The process will take longer if the colour of cover image is very diverse.

5. Create a stego-key

Stego-key is used to extract the secret image. In this proposed algorithm, the stego-key is determined by counting the weight and height of the secret image. Moreover, the stego-key is also used to validate the size of message container area.

2.2. Message extraction method

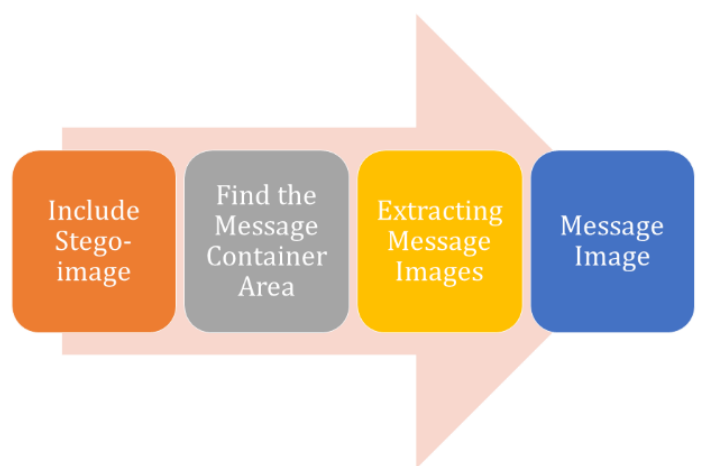

Fig. 3. Process of message extraction 
After the hidden message is successfully embedded in the image, the message will be sent to the recipient, who extracts the message. Fig. 3 shows the process of message extraction from a stego-image.

The following steps describe the Message Extraction Process:

1. Include stego image

Extraction step starts with scanning the stego-image and check whether the width of the image is divisible by 8 or not. If it is indivisible by 8 , the algorithm will declare two variables: id $x$ and id $y$, that can determine the starting point to read the image.

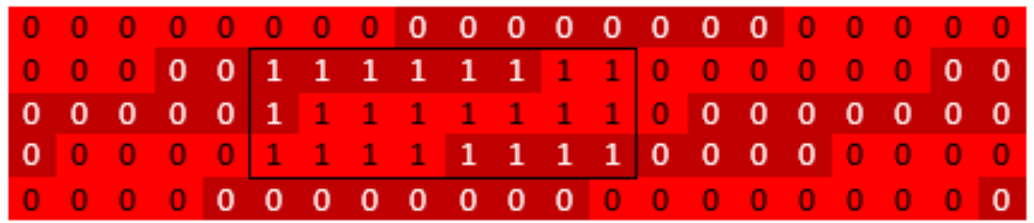

Fig. 4. Length of stego-bit indivisible by 8

Appertaining to Fig. 4, if the stego-image length is indivisible by 8 , the algorithm generates new binary values. The position of the hidden message is at the center, surrounded by a black border. The next equation is to acquire the id $x$ value after calculating the image length:

$$
\begin{aligned}
& \mathrm{id} x=w \bmod (8), \\
& \mathrm{id} x=21 \bmod (8), \\
& \mathrm{id} x=5 .
\end{aligned}
$$

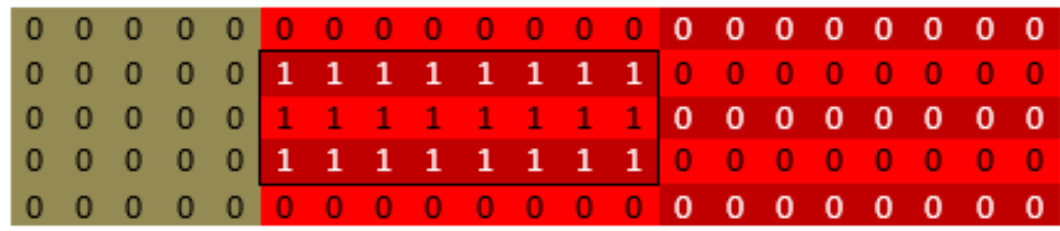

Fig. 5. Bit-reading after idx value determination

Fig. 5 shows that the bit reading starts from the 6th bit. Index array in programming begins from 0 ; thus idx value of 5 implies that the bit-reading begins from the 6th bit. By this calculation and bit-reading, stego-image extraction will conduct successfully on a cropped image.

2. Find the message container area

After scanning the stego-image, stego-key should be validated. The use of stegokey is to ensure that the extracted message has the same size as the secret image. The algorithm only scans the area with "1" values, because the area with value "0" are beyond the message container area.

3. Extracting message images

After finding the message container area and stego-key is validated, the extraction process will take place to form the original/secret image by changing binary into pixel. 


\section{Result}

\subsection{Implementation CEPP Algorithm}

The message embedding process is conducted in Python programming language as shown in Fig. 1. The following is a snippet of source code for processing the cover image and secret image.

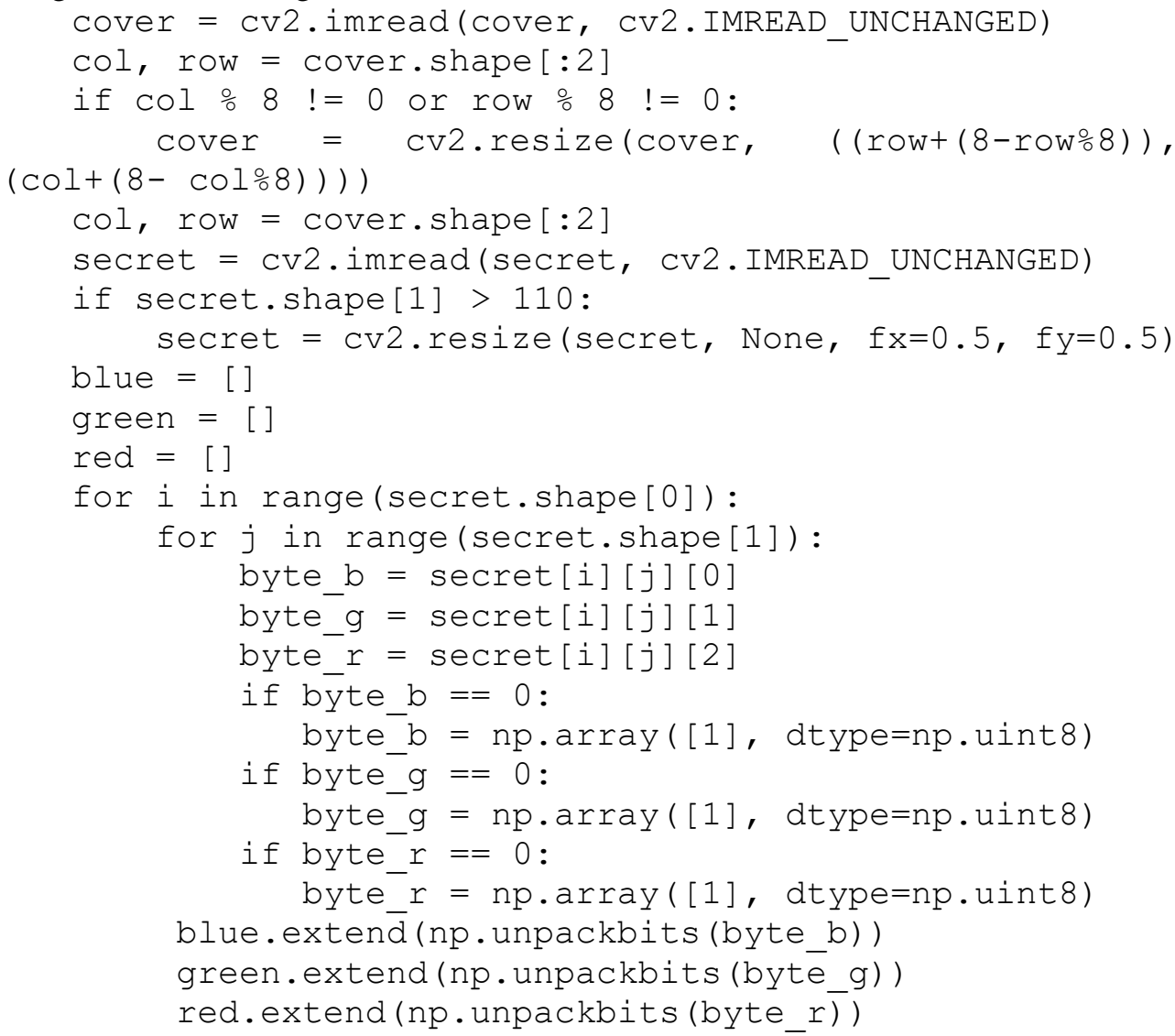

The secret image is transformed into a list, and then the algorithm calculates the container coordinates to insert the message into the cover image. Calculating the container coordinates, cover image validation, and message length towards container size validation are several processes. The source code used is as follows:

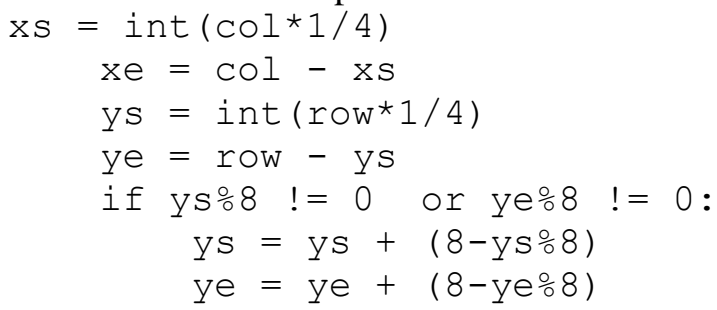


3.2. Message embedding algorithm and create a stego-key

The following Source Code, is a snippet of the Embedded Center Positioning algorithm's core program, the message embedding process to the container area and creating the stego-key.

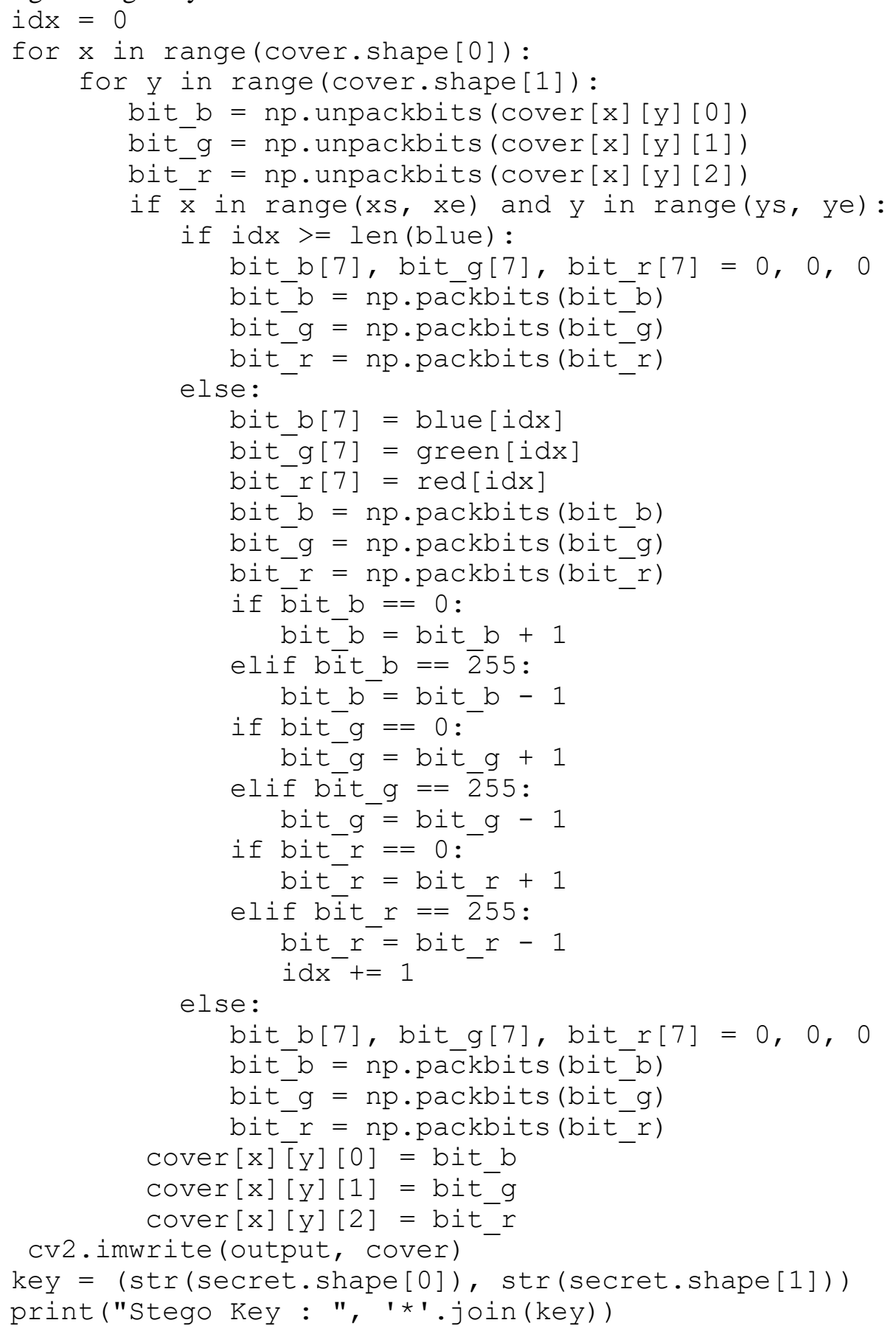




\subsection{Message extraction algorithm}

Before extracting the hidden message from stego-image, the image is read by the program using the LoadImage Function and DecKey Function to read stego-key with the following source code:

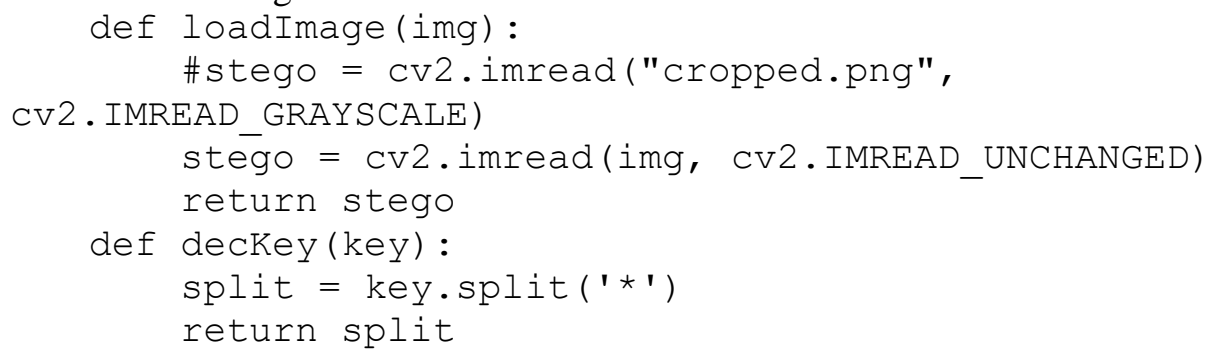

The following is the main source code for the message extraction process:

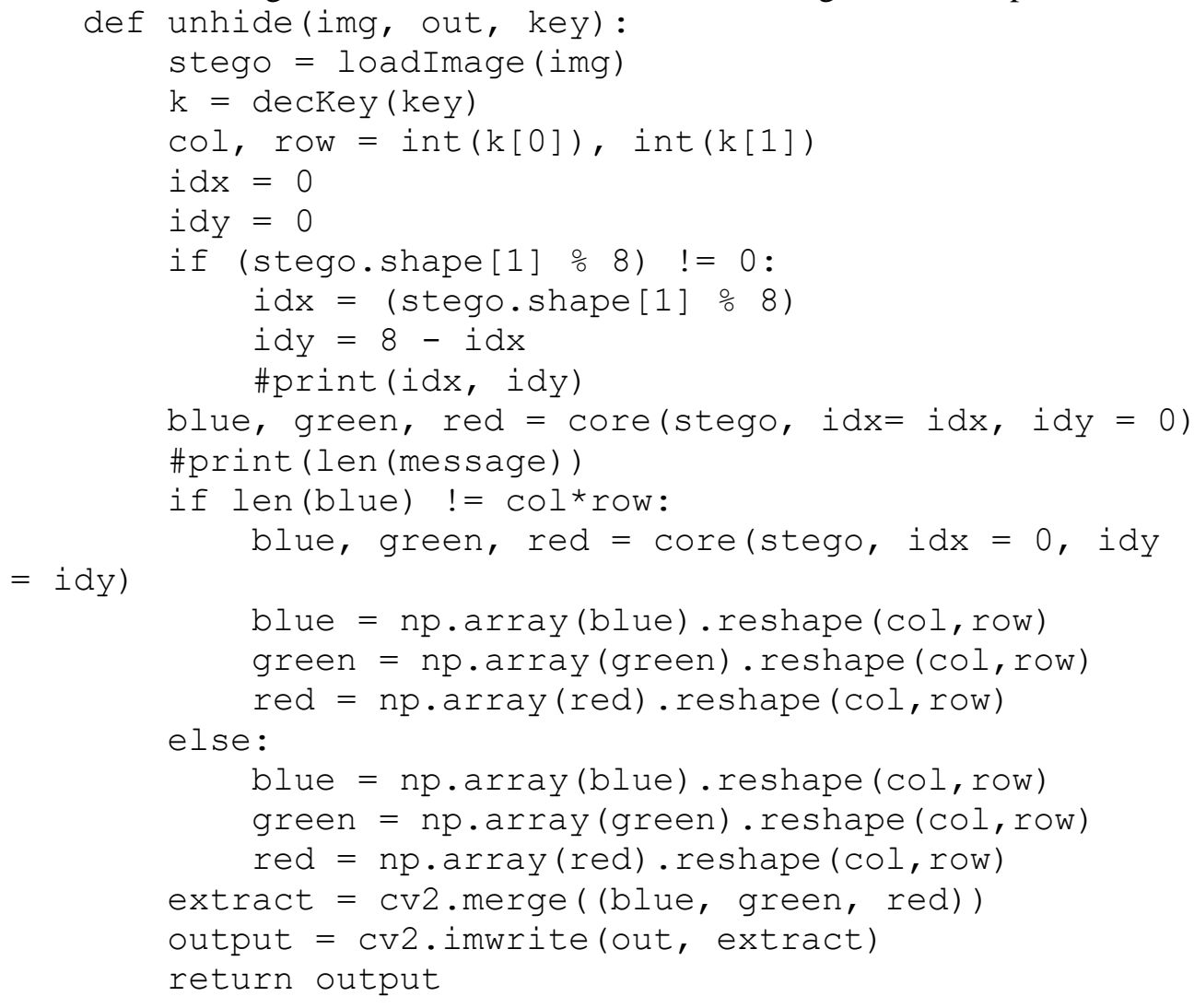

\section{Discussion}

4.1. embedding message testing

Test results in the embedding process determine the success rate of the program. Several PNG formatted images are used as cover images, all are RGB as presented in Fig. 6. 

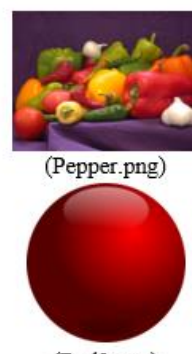

(Red2.png)

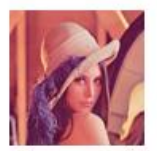

(Lenna.png)

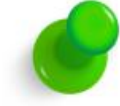

(Green.png)

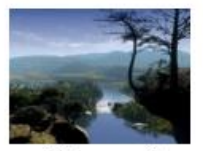

(Nature.png)

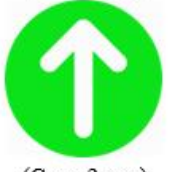

(Green2.png)

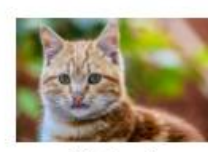

(Cat.png)

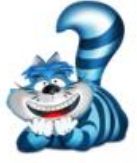

(Blue.png)

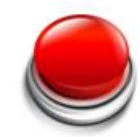

(Red.png)

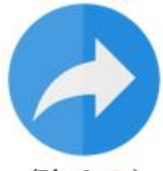

(Blue2.png)

Fig. 6. Cover image

Selected cover images must vary in color combination, with some samples they are considerably dominant in the color of Red, Blue, or Green (RGB). With varying the color, it will also widen the color spectrum of sample images to analyze the change in image size and quality between the cover image and the stego-image.

The quality of the stego-image is heavily influenced by the size of the secret image. The smaller the secret image while the cover image is bigger will cause the proportion of cropping area to increase. The amount of the allowable pixel value of the secret image can be calculated using the next equation:

$$
\text { cover image size }=w h \text {, }
$$

$$
\begin{aligned}
& \text { message container size }=\frac{1}{2} w \frac{1}{2} y=\frac{1}{4} w h, \\
& \text { LSB that can be used }=\frac{\frac{1}{4} w h}{8}=\frac{1}{32} w h=\frac{w h}{32} .
\end{aligned}
$$

Results of the embedding process are given in Table 1. All cover images are listed in Fig. 6, whereas the message image inserted into the cover image is a 3.44 KB PNG image with a dimension of $45 \times 80$ pixels, being much smaller than each cover images.

Table 1. Steganography testing

\begin{tabular}{|c|c|c|c|c|}
\hline Cover image & $\begin{array}{c}\text { Cover image } \\
\text { dimension }\end{array}$ & $\begin{array}{c}\text { Cover image } \\
\text { size }(\mathrm{KB})\end{array}$ & $\begin{array}{c}\text { Stego-image } \\
\text { size }\end{array}$ & $\begin{array}{c}\text { Stego-image } \\
\text { dimension }\end{array}$ \\
\hline Pepper.png & $512 \times 384$ & 280 & 260 & $512 \times 384$ \\
\hline Lenna.png & $512 \times 512$ & 462 & 422 & $512 \times 512$ \\
\hline Nature.png & $400 \times 300$ & 162 & 152 & $400 \times 300$ \\
\hline Cat.png & $600 \times 352$ & 500 & 480 & $600 \times 352$ \\
\hline Red.png & $512 \times 512$ & 45 & 80 & $512 \times 512$ \\
\hline Red2.png & $512 \times 512$ & 37 & 50 & $512 \times 512$ \\
\hline Green.png & $512 \times 512$ & 90 & 120 & $512 \times 512$ \\
\hline Green2.png & $512 \times 512$ & 21 & 40 & $512 \times 512$ \\
\hline Blue.png & $512 \times 512$ & 266 & 280 & $512 \times 512$ \\
\hline Blue2.png & $512 \times 512$ & 19 & 32 & $512 \times 512$ \\
\hline
\end{tabular}

Even though initially, the cover and message image's size and dimension are different, Table 1 and Fig. 7 show that the stego-image resulted in having precisely the same dimension as the cover image. By equalling the image's dimension, the quality testing measured using MSE (Mean Square Error) and PSNR is expected to show a better result. 


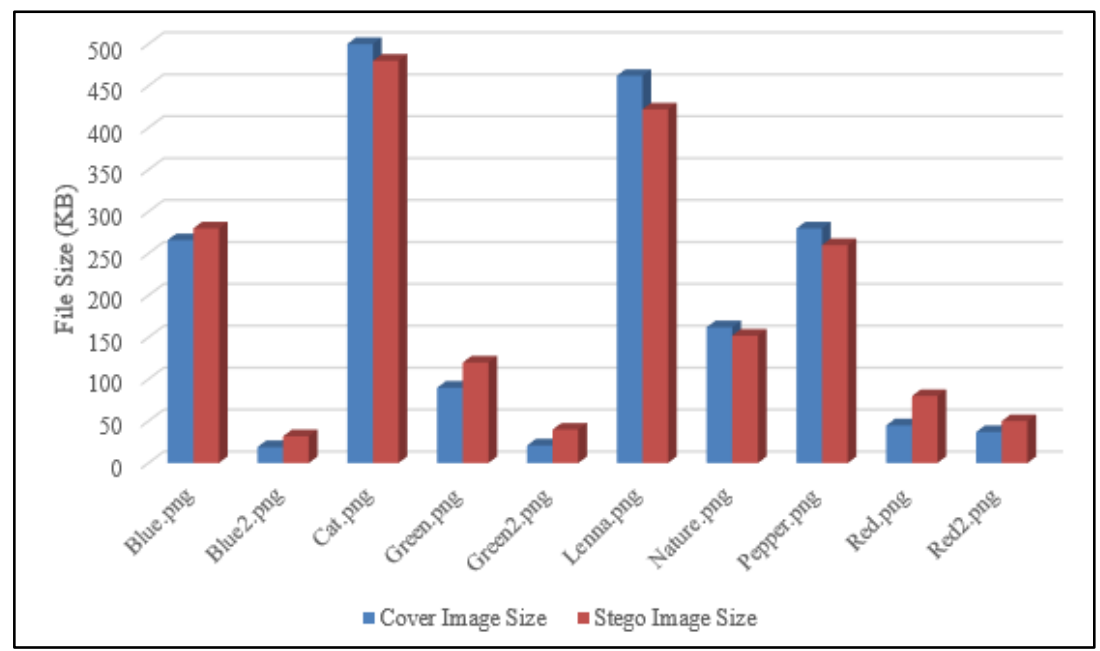

Fig. 7. Visualization of steganography testing

However, the program was unable to maintain image's size due to the compression process of the stego-image, converting it into a PNG image format. Despite its change in size, since the lossless compression method is applied, there is only a slight effect on the image quality. Based on the size, stego-images generated from the program are categorized into two: decrement or increment in size. There is a tendency that images with a wide variety of colors (e.g., cat.png, lenna.png, nature.png) have a smaller stego-image file size, whereas images having one dominant color (e.g., red.png, green.png, blue.png) indicate the opposite.

\subsection{Extraction message testing}

To start the message extraction process, the program will need a stego-key. Stegokey is responsible for matching the original message image's size and the size of the message image in the message container area. The method proposed in this algorithm is that during the message insertion process, the final bits in the message container area are assigned to " 1 ", while in areas outside the container, the bits are assigned to " 0 ". For each side of the rectangular area, bits are also assigned to " 1 " as a boundary. Determining the boundary area is necessary so that the algorithm can read the coordinates of the message container area during the message extraction process and then adjust it to the message's length and width based on the previously entered stegokey.

This study applies the LSB matching method, with bits outside the container boundary being replaced with " 0 " to simplify the extraction process. In Fig. 8, the process of reading a message container area is illustrated by marking the message bit as " 1 " in the middle area and on each side of the container. In contrast, the remaining bits outside the container area are marked as " 0 ". By implementing this method, when the image is cropped, whether symmetrical or asymmetrical, the messages in the container area can still be extracted. However, if the cropping reaches the container area's boundary, the message will still be corrupted. 


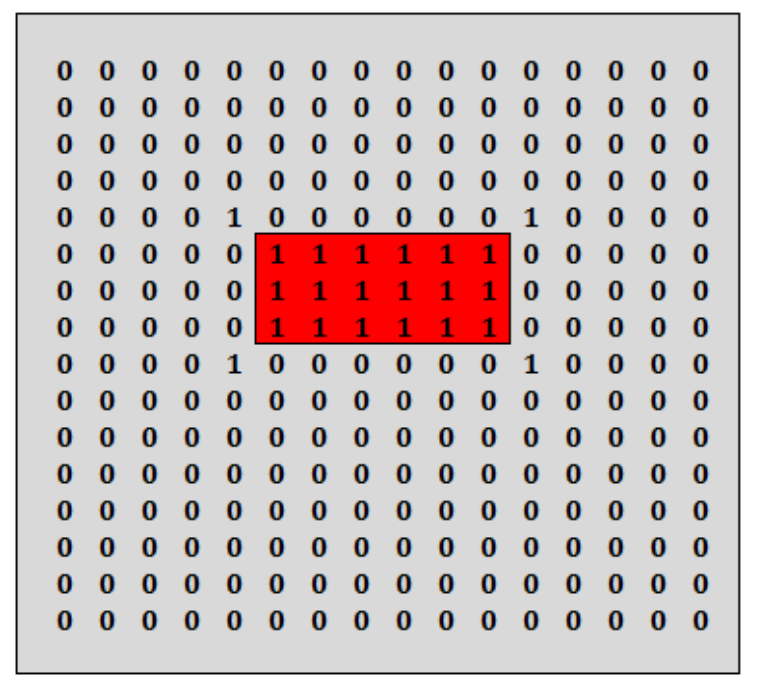

Fig. 8. Process of reading the message container area

\subsection{Imperceptibility test}

Imperceptibility testing aims to see how difficult or easy stego-images can be detected by human vision or the Human Visual System (HVS). This test was carried out manually by involving 35 respondents who were asked to fill in the questionnaire by comparing original images (cover images) as well as images with secret images (stego-images). The questionnaire includes several sample images, as given in Table 2.

Table 2. Imperceptibility test result

\begin{tabular}{|l|c|c|c|}
\hline Stego image & Different & Slightly different & No different \\
\hline Pepper.png & 2 & 2 & 31 \\
\hline Lenna.png & 1 & 1 & 33 \\
\hline Nature.png & 1 & 2 & 32 \\
\hline Cat.png & 0 & 3 & 32 \\
\hline Red.png & 1 & 3 & 31 \\
\hline Red2.png & 1 & 2 & 32 \\
\hline Green.png & 2 & 3 & 30 \\
\hline Green2.png & 1 & 5 & 29 \\
\hline Blue.png & 0 & 3 & 32 \\
\hline Blue2.png & 1 & 3 & 31 \\
\hline
\end{tabular}

Imperceptibility test includes five image samples, which compares the cover image and stego image. Based on Table 2 and Fig. 9, around 89\% of respondents stated there is no difference between the cover image and the stego image. It can be concluded that there is no significant change resulted from the steganography process. 


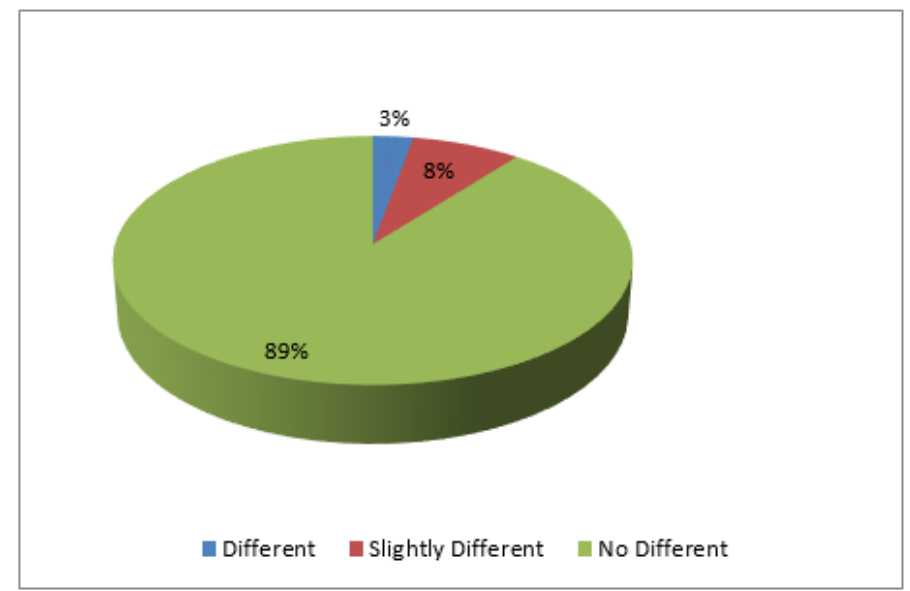

Fig. 9. Visualization of imperceptibility test result

\subsection{Fidelity testing}

Fidelity test aims to see the quality of the stego-image, whether a significant change exists after the message is embedded. The test is performed by calculating the MSE and PSNR. Results from this test are given in Table 3.

Tabel 3. Result of fidelity testing

\begin{tabular}{|c|c|c|c|c|c|}
\hline Cover image & Cover image size (KB) & $\begin{array}{c}\text { Message image } \\
\text { size (KB) }\end{array}$ & $\begin{array}{c}\text { Stego-image } \\
\text { Size }\end{array}$ & MSE & PSNR \\
\hline Pepper.png & 280 & 3.44 & 260 & 0.5 & 51.08 \\
\hline Lenna.png & 462 & 3.44 & 422 & 0.5 & 51.08 \\
\hline Nature.png & 162 & 3.44 & 152 & 0.4 & 52.9 \\
\hline Cat.png & 500 & 3.44 & 480 & 0.4 & 52.25 \\
\hline Red.png & 45 & 3.44 & 80 & 0.47 & 51.35 \\
\hline Red2.png & 37 & 3.44 & 50 & 0.4 & 52.01 \\
\hline Green.png & 90 & 3.44 & 120 & 0.66 & 49.87 \\
\hline Green2.png & 21 & 3.44 & 40 & 0.54 & 50.75 \\
\hline Blue.png & 266 & 3.44 & 280 & 0.63 & 50.09 \\
\hline Blue2.png & 19 & 3.44 & 32 & 0.39 & 52.18 \\
\hline
\end{tabular}

High PSNR value denotes a good image quality. Table 3 shows the PSNR value between the cover image and the stego-image is very good, reaching $52.9 \mathrm{~dB}$, exceeding the standard $40 \mathrm{~dB}$. On the other hand, the value of MSE is between 0.6, implying that changes between the cover image and stego-image are not significant [18, 20-24]. Visualization of the results of the MSE and PSNR test is provided in Figs 10 and 11.

Figs 10 and 11 prove an increase in image quality compared to previous studies. Research by Swain [18] has an average PSNR value of $47 \mathrm{~dB}$, whereas the CEPP algorithm proposed in this work is around $52 \mathrm{~dB}$. 


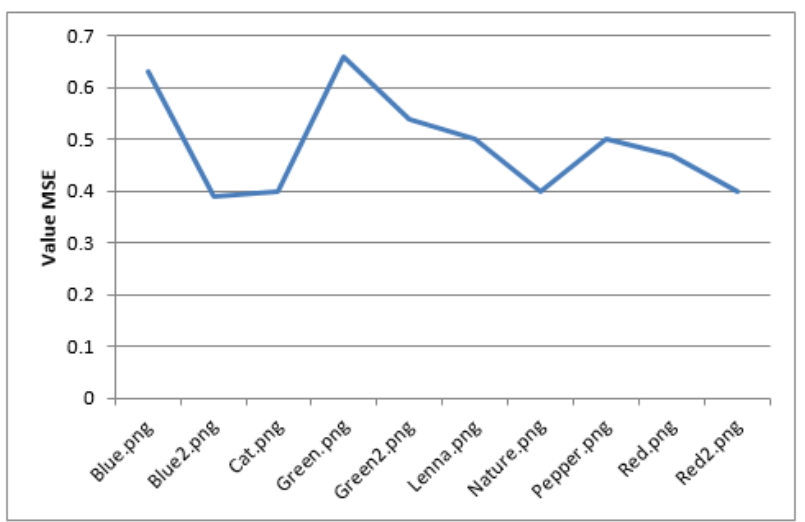

Fig. 10. Visualization of MSE result

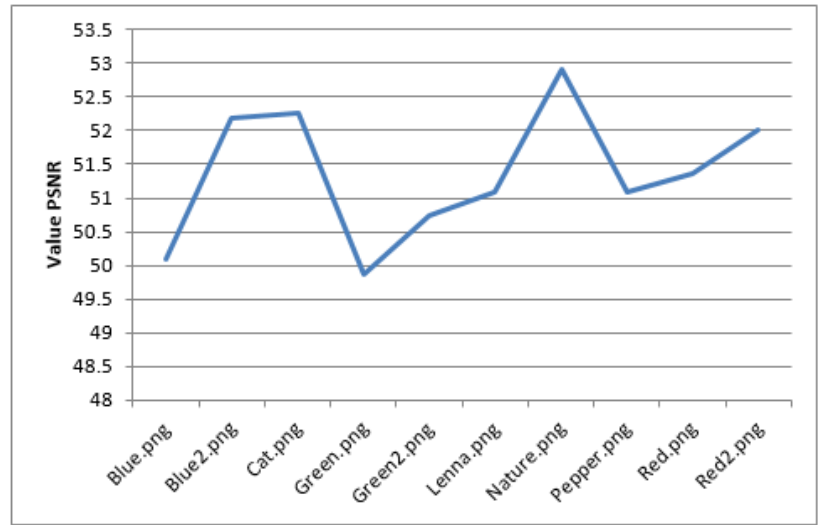

Fig. 11. Visualization of PSNR result

\subsection{Robustness test}

A robustness test is conducted to observe whether the stego-image can resist image processing (specifically cropping) attacks and extract the secret images. In the first test, we symmetrically crop the stego-image in several directions with the results presented in Table 4 ( $\checkmark$ is the extraction success; $X$ is the extraction fail).

According to the test results, the stego-image can be cropped symmetrically from all four directions but with limitations. For the left, right, and upper side, we can crop the stego-image at a maximum of $25 \%$; otherwise, the message cannot be extracted. The extraction process will fail due to the embedding process that takes 0.25 of the image's length or width as the message container area's boundary, making the image resistance is only $25 \%$ of the overall image. Cropping from the lower side shows that the message can still be extracted almost $70 \%$ from that side (only one empirical case). In determining the starting point for the message container's boundary, it does not include the lower side of the image. Therefore, the extraction process will only fail if we crop the lower side by more than $70 \%$. 
Table 4. Crop test (symmetry)

\begin{tabular}{|c|c|c|c|c|c|c|c|c|c|c|c|c|c|c|c|}
\hline \multirow{2}{*}{ No } & \multirow{2}{*}{$\begin{array}{l}\text { Cover } \\
\text { image }\end{array}$} & \multirow{2}{*}{$\begin{array}{c}\text { Message } \\
\text { image } \\
\end{array}$} & \multirow{2}{*}{$\begin{array}{c}\text { Crop } \\
\text { direction }\end{array}$} & \multicolumn{12}{|c|}{ Crop percentage } \\
\hline & & & & 20 & 25 & 30 & 35 & 40 & 45 & 50 & 55 & 60 & 65 & 70 & 75 \\
\hline \multirow{4}{*}{1} & \multirow{8}{*}{$512 \times 512$} & \multirow{4}{*}{$45 \times 45$} & Left & $\checkmark$ & $\checkmark$ & $x$ & $x$ & $x$ & $x$ & $x$ & $x$ & $x$ & $x$ & $x$ & $x$ \\
\hline & & & Right & $\checkmark$ & $\checkmark$ & $x$ & $x$ & $x$ & $x$ & $x$ & $x$ & $x$ & $x$ & $x$ & $x$ \\
\hline & & & $\mathrm{Up}$ & $\checkmark$ & $\checkmark$ & $x$ & $x$ & $x$ & $x$ & $x$ & $x$ & $x$ & $x$ & $x$ & $x$ \\
\hline & & & Down & $\checkmark$ & $\checkmark$ & $\checkmark$ & $\checkmark$ & $\checkmark$ & $\checkmark$ & $\checkmark$ & $\checkmark$ & $\checkmark$ & $x$ & $x$ & $x$ \\
\hline \multirow{4}{*}{2} & & \multirow{4}{*}{$90 \times 90$} & Left & $\checkmark$ & $x$ & $x$ & $x$ & $x$ & $x$ & $x$ & $x$ & $x$ & $x$ & $x$ & $x$ \\
\hline & & & Right & $\checkmark$ & $x$ & $x$ & $x$ & $x$ & $x$ & $x$ & $x$ & $x$ & $x$ & $x$ & $x$ \\
\hline & & & $\mathrm{Up}$ & $\checkmark$ & $x$ & $x$ & $x$ & $x$ & $x$ & $x$ & $x$ & $x$ & $x$ & $x$ & $x$ \\
\hline & & & Down & $\checkmark$ & $\checkmark$ & $x$ & $x$ & $x$ & $x$ & $x$ & $x$ & $x$ & $x$ & $x$ & $x$ \\
\hline \multirow{4}{*}{3} & \multirow{8}{*}{$1500 \times 1500$} & \multirow{4}{*}{$45 \times 45$} & Left & $\checkmark$ & $\checkmark$ & $x$ & $x$ & $x$ & $x$ & $x$ & $x$ & $x$ & $x$ & $x$ & $x$ \\
\hline & & & Right & $\checkmark$ & $\checkmark$ & $x$ & $x$ & $x$ & $x$ & $x$ & $x$ & $x$ & $x$ & $x$ & $x$ \\
\hline & & & Up & $\checkmark$ & $\checkmark$ & $x$ & $x$ & $x$ & $x$ & $x$ & $x$ & $x$ & $x$ & $x$ & $x$ \\
\hline & & & Down & $\checkmark$ & $\sqrt{ }$ & $\checkmark$ & $\checkmark$ & $\checkmark$ & $\sqrt{ }$ & $\checkmark$ & $\checkmark$ & $\checkmark$ & $\checkmark$ & $\checkmark$ & $x$ \\
\hline \multirow{4}{*}{4} & & \multirow{4}{*}{$90 \times 90$} & Left & $\checkmark$ & $\checkmark$ & $x$ & $x$ & $x$ & $x$ & $x$ & $x$ & $x$ & $x$ & $x$ & $x$ \\
\hline & & & Right & $\checkmark$ & $\checkmark$ & $x$ & $x$ & $x$ & $x$ & $x$ & $x$ & $x$ & $x$ & $x$ & $x$ \\
\hline & & & Up & $\checkmark$ & $\checkmark$ & $x$ & $x$ & $x$ & $x$ & $x$ & $x$ & $x$ & $x$ & $x$ & $x$ \\
\hline & & & Down & $\checkmark$ & $\sqrt{ }$ & $\sqrt{ }$ & $\checkmark$ & $\bar{\checkmark}$ & $\checkmark$ & $\checkmark$ & $\checkmark$ & $\checkmark$ & $\checkmark$ & $x$ & $x$ \\
\hline
\end{tabular}

The next observation is by asymmetrically cropping the stego-image. In this test, the stego-image is cropped from several sides with different cropping percentages ranging from 5 to $50 \%$. The cropping process is done sequentially from left, right, up, and bottom. Suppose the stego-image is firstly cropped from the left as much as $5 \%$. The resulting image is then cropped back from the right side for about $10 \%$, and so on. Test results of the asymmetrical cropping on stego-images are presented in Table 5 ( $\checkmark$ is the extraction success; $X$ is the extraction fail).

Table 5. Crop test (asymmetry)

\begin{tabular}{|c|c|c|c|c|c|}
\hline \multirow{2}{*}{ No } & \multicolumn{4}{|c|}{ Crop percentage } & \multirow{2}{*}{ Message extraction } \\
\hline & Left & Right & Up & Down & \\
\hline 1 & 10 & 15 & 0 & 0 & $\checkmark$ \\
\hline 2 & 15 & 10 & 0 & 0 & $\checkmark$ \\
\hline 3 & 10 & 15 & 5 & 0 & $x$ \\
\hline 4 & 10 & 15 & 0 & 5 & $x$ \\
\hline 5 & 0 & 0 & 10 & 15 & $\checkmark$ \\
\hline 6 & 0 & 0 & 15 & 10 & $\checkmark$ \\
\hline 7 & 0 & 5 & 10 & 15 & $x$ \\
\hline 8 & 0 & 0 & 10 & 15 & $\checkmark$ \\
\hline 9 & 25 & 20 & 0 & 0 & $x$ \\
\hline 10 & 25 & 0 & 0 & 45 & $\checkmark$ \\
\hline 11 & 0 & 25 & 0 & 45 & $\checkmark$ \\
\hline 12 & 0 & 0 & 25 & 0 & $\checkmark$ \\
\hline 13 & 25 & 0 & 0 & 50 & $x$ \\
\hline 14 & 0 & 25 & 0 & 50 & $x$ \\
\hline 15 & 0 & 0 & 25 & 50 & $x$ \\
\hline
\end{tabular}


Asymmetrically cropping the stego-image was carried out 15 times with the results available in Table 5. Test results show that asymmetrical cropping can be performed effectively only if the image is cropped on two sides. From Table 5, we can see that test cases number $1,2,5,6,8,10,11$, and 12 are only cropped on two sides; thus, messages can be extracted successfully. However, when the cropping attack is performed on more than two sides, such as in test cases number 3, 4, and 7, the extraction process will fail. The message container are damaged when the third cropping is conducted on a different side.

Results of observations on test cases number 10,11 , and 12 prove that the message can still be extracted if the maximum cropping area at the bottom is $45 \%$ and 25\% from either left, right, or top. Meanwhile, test cases number 13, 14, and 15 fail to extract since the total cropping areas are greater than $70 \%$.

\section{Conclusion}

Based on the discussion, the CEPP algorithm shows a remarkable result in image steganography, proved by its success in the embedding and message extraction processes. Stego-images generated using the CEPP algorithm are considered very good, as indicated by the MSE and PSNR values. The images can also be received through the Human Visual System (HVS), shown by the Imperceptibility Test results. CEPP algorithm can resist cropping attacks symmetrically from the left, right, and top of a maximum to $25 \%$, while performing better at the bottom with a resistance limit of more than $70 \%$. This proposed algorithm can also resist asymmetric cropping attacks as long as they are only performed on two sides with a total cropping percentage, not more than $25 \%$. All results are based on empirical data.

Acknowledgments: This research is funded by The Ministry of Research and Technology/The National Research and Innovation Agency, the Republic of Indonesia under the schema Doctoral Research Grant 2021.

\section{References}

1. D e s o k y, A. Noiseless Steganography. CRC Press, 2012.

2. A bd E1-Latif, A. A., B. Abd El-A tt, S. E. Venega s-A nd raca. A Novel Image Steganography Technique Based on Quantum Substitution Boxes. - Optics and Laser Technology, Vol. 116, 2019, pp. 92-102.

3. A 1-A f a ndy, K. A., O. S. F a ragallah, A. E $1 \mathrm{mhal}$ a w y, E. S. M. E 1-R abai e, G. M. E 1-B a n b y. High Security Data Hiding Using Image Cropping and LSB Least Significant Bit Steganography. - In: 4th International Colloquium on Information Science and Technology (CIST'16), IEEE, 2016, pp. 400-404.

4. K u m a r, V., D. K u m a r. Digital Image Steganography Based on Combination of DCT and DWT. - Communications in Computer and Information Science, Vol. 101, 2010, pp. 596-601.

5. Ku mar, S. K., P. D. K. R edd y, G. R a m e s h, V. R. M a d d u m la. Image Transformation Technique Using Steganography Methods Using LWT Technique. - International Information and Engineering Technology Assocation, Vol. 36, 2019, No 3, pp. 233-237.

6. J u a r e z-S and ov a 1, O., M. C e d i 11 o-H e rn and e z, G. S a n c h e z-P e r e z, K. T o s c a n o$\mathrm{Me}$ d in a, H. Pe re z-M e an a, M. N a k a n o-M i y a t a ke. Compact Image Steganalysis for LSB-Matching Steganography. - In: Proc. of 5th International Workshop on Biometrics and Forensics, IWBF, 2017. 
7. K a d h i m, I. J., P. Pr e m a r a t n e, P. J. V i a l, B. H a 11 or a n. Comprehensive Survey of Image Steganography: Techniques, Evaluations, and Trends in Future Research. - Neurocomputing, Vol. 335, 2019, pp. 299-326.

8. Mishra, M., P. Mishra, M. C. Adhikary. Digital Image Data Hiding Techniques: A Comparative Study. - Ansvesa, Vol. 7, 2014, No 2, pp. 105-115.

9. M i s h r a, B., R. B e g, V. P. S ing h. Information Security through Digital Image Steganography Using Multilevel and Compression Technique. - MIT International Journal of Computer Science \& Information Technology, Vol. 3, 2013, No 1, pp. 26-29.

10. H u, D., L. W a n g, W. J i a n g, S. Z h e n g, B. L i. A Novel Image Steganography Method via Deep Convolutional Generative Adversarial Networks. - IEEE Transactions on Information Forensics and Security, Vol. 6, 2018, pp. 38303-38314.

11. S in g h, S., R. B e g, T. J. S i d d i qu i. Robust Image Steganography Using Complex Wavelet Transform. - In: Proc. of International Multimedia, Signal Processing and Communication Technologies, IMPACT, 2013, pp. 56-30.

12. Hu s sain, M., A. W. A. W a hab, Y. I. B in Idris, A. T. S. Ho, T. S. Jung. Image Steganography in Spatial Domain: A Survey. - Signal Processing: Image Communication Vol. 65, 2018, pp. 46-66.

13. Z h o u, Z., Y. M u, Q. M. J. W u. Coverless Image Steganography Using Partial-Duplicate Image Retrieval. - Soft Computing, Vol. 23, 2019, No 23, pp. 4927-4938.

14. D a rwis, D., A. J u n a id i, W a milian a. A New Approach of Steganography Using Center Sequential Technique. - In: Journal of Physics: Conference Series. Vol. 1338. 2019.

15. Ju are z-S andoval, O., A. Fierro-Radilla, A. Espejel-Trujillo, M. Nakan o$\mathrm{M}$ i y a t a ke, H. P e r e z-M e a n a. Cropping and Noise Resilient Steganography Algorithm Using Secret Image Sharing. - In: Proc. of 6th International Conference on Graphic and Image Processing (ICGIP'14), Vol. 9443, 2015.

16. A k a r, F., Y. Y a $1 \mathrm{~m}$ a n, H. S. V a r o l. Data Hiding in Digital Images Using a Partial Optimization Technique Based on the Classical LSB Method. - Turkish Journal of Electrical Engineering and Computer Sciences, Vol. 21, 2013, pp. 2037-2047.

17. S e t i a d i, D. R. I. M., J. J u m a n t o. An Enhanced LSB-Image Steganography Using the Hybrid Canny-Sobel Edge Detection. - Cybernetics and Information Technologies, Vol. 18, 2018, No 2, pp. 74-88.

18. S w a i n, G., S. K. L e n k a. A Novel Steganography Technique by Mapping Words with LSB Array. - International Journal of Signal and Imaging Systems Engineering, Vol. 8, 2015, No 1-2, pp. 115-122.

19. A 1 h u m y a n i, H. Efficient Image Cipher Based on Baker Map in the Discrete Cosine Transform. - Cybernetics and Information Technologies, Vol. 20, 2020, No 1, pp. 68-81.

20. W a mili a n a, M. U s m a n, A. Hi jri a n i, W ar s it o, R. S e t i a w a n. The Hybrid Methods of Column Transposition with Adaptive Minimum Error Least Significant Bit Replacement (AMELSBR) Using File jpg/jpeg and png. - International Journal of Computer Science and Network Security, Vol. 17, 2017, No 7, pp. 174-179.

21. Z h a n g, Y., C. Q i n, W. Z h a n g, F. L i u, X. L u o. On the Fault-Tolerant Performance for a Class of Robust Image Steganography. - Signal Processing, Vol. 146, 2018, pp. 99-111.

22. Z h a n g, X., F. P e n g, M. L o n g. Robust Coverless Image Steganography Based on DCT and LDA Topic Classification. - IEEE Transactions on Multimedia. Vol. 20, 2018, No 12, pp. 3223-3238.

23. W u, P., Y. Y a n g, X. L i. StegNet: Mega Image Steganography Capacity with Deep Convolutional Network. - Future Internet, Vol. 10, 2018, pp. 1-15.

24. S a hu, A. K., G. S w a i n, E. S. B a b u. Digital Image Steganography Using Bit Flipping. Cybernetics and Information Technologies, Vol. 18, 2018, No 1, pp. 69-80.

Received: 15.12.2020; Second Version: 17.03.2021; Third Version: 13.04.2021; Accepted: 28.04.2021 (fast track) 